\title{
Examination of How Size-Effect Modifies the Stiffness and Mass Matrices of Nanotrusses/Nanoframes
}

\author{
Hayri Metin Numanoğlu \\ ${ }^{a}$ Giresun University, Civil Engineering Department, Division of Mechanics, Giresun, Turkey \\ *E-mail address: metin_numanoglu@ hotmail.com \\ ORCID number of author: \\ 0000-0003-0556-7850
}

\begin{abstract}
The effect of the nonlocal parameter on the free vibration analysis of nano scaled trusses and frames is examined. Accordingly, firstly, the axial and bending vibrations of the nano scaled longitudinal element are formulated. Simple rod and Euler-Bernoulli assumptions are considered for axial and bending vibrations, respectively. Finite element matrices are obtained by applying the average weighted residue to the nonlocal equation of motion for free vibration. These matrices are combined according to the freedoms of longitudinal element and the matrix displacement method is explained for structures consisting of discrete longitudinal elements. It is discussed how the classical stiffness and mass matrices are modified by the atomic parameter.
\end{abstract}

Keywords: Mass matrix, matrix displacement method, nanoframe, nanotruss, nonlocal free vibration, stiffness matrix.

\section{Introduction}

The fact that nano scaled materials play a role in today's modern applications such as biosensors, resonators, transistors, gas sensors, nanocantilever, microcircuits, medicine, and dental has increased the importance of investigation on their mechanical properties as well as the physical, electrical, optical, and thermal properties of these materials. One-dimensional materials such as carbon nanotubes, boron nitride nanotubes, silica carbide nanotubes, metallic nanowires, and two-dimensional materials such as graphene, borophene, silicene are encountered in today's modern applications. In the investigation of mechanical behavior of such materials, because of expensiveness, high specialization requirement, high computational volume, long and inefficient processes, the studies based molecular dynamics simulation have led researchers to use mechanical structure models known from solid mechanics. It is a well-known result of scientific studies that models based classical physics theories do not give accurate results. Therefore, researchers have efforted to explain the mechanical behavior of nano scaled structures by combining these models with higher-order continuum formulations such as nonlocal elasticity, modified couple stress elasticity, modified strain gradient elasticity, doublet mechanics, surface energy.

When studies on the mechanics of nano scaled structures are examined, it can be observed that the nonlocal elasticity theory has been investigated more intensively than other higher-order continuum mechanics theories. Mechanical structure models such as rod [1-11] and beam [1225] form the basis of studies in the nonlocal mechanics of nanostructures. Also, recently, studies 
that mention mechanical analyses with nonlocal elasticity of discrete structures such as truss and frame [26-28] have entered the scientific literature.

Analytical methods such as double integration, separation of variables and series expansion have been able to solve some problems in solid mechanics [29-34]. However, reasons such as the inclusion of some parameters (elastic foundation/medium, thermal/hygrothermal environment, electro-magnetic environment, functionally grading, etc.) to the problem or the complication of boundary conditions may make the using of analytical methods in the problem impossible. Therefore, the using of numerical methods has gained importance in the solution of solid mechanics problems [35-39]. Moreover, the using of the finite element method in solid mechanics problems involving nonlocal elasticity is available in the scientific literature [2$4,10,11,17-26,40,41]$.

How the nonlocal parameter affects the classical elasticity solution in mechanical analysis of nano scaled truss and frame structures is discussed in this current study. It is planned that to scrutinize the nonlocal parameter on the matrices in the study [26] where vibration analyses of nanotrusses and nanoframes were given in detail. Firstly, the stiffness and mass matrices of nonlocal axial and bending vibrations of nano scaled longitudinal element are obtained using the weighted residue method. Then, stiffness and mass matrices are presented for nonlocal free vibration analysis of discrete structural models formed by axial or bending members by matrix displacement method. Finally, the effect of nonlocal parameter on the matrices of nanostructure is discussed.

\section{Nonlocal Equation of Motions and Application of Average Weighted Residue}

For vibration analysis of discrete structures consisting of nano scaled longitudinal elements, firstly, the axial and bending vibrations of the longitudinal element should be investigated. The free vibration equations are solved by the average weighted residue defined below:

$$
\mathrm{I}=\int_{0}^{L} h \cdot R \mathrm{~d} x
$$

where I, $h$, and $R$ define the average weighted residue, weighting function, and residue, respectively. $L$ is length of the longitudinal element. According to method, the average weighted residue should be equal to 0 . The residue is the equation to be solved, namely, the equation of motion. On the other hand, weighting function is $h=\phi^{\mathrm{T}}$ and $\phi$ is a shape function. The shape functions are written as follow for axial and bending vibrations, respectively:

$$
\begin{gathered}
\phi_{a}=\{1-\xi \quad \xi\} \\
\phi_{b}=\left\{1-3 \xi^{2}+2 \xi^{3} \quad L\left(-\xi-2 \xi^{2}+\xi^{3}\right) \quad 3 \xi^{2}-2 \xi^{3} \quad L\left(-\xi^{2}+\xi^{3}\right)\right\}
\end{gathered}
$$

where $\xi=x / L$ is called as nondimensional longitudinal coordinate.

\subsection{Axial Vibration}

The equation of motion of nonlocal free axial vibration for nano scaled structures according to the simple (without shear effect) rod formulation is as [3,30]: 


$$
E A \frac{\partial^{2} u}{\partial x^{2}}-\rho A \frac{\partial^{2} u}{\partial t^{2}}+\left(e_{0} a\right)^{2} \rho A \frac{\partial^{4} u}{\partial x^{2} \partial t^{2}}=0
$$

where $E, A$, and $\rho$ state the modulus of elasticity, area of cross-section, and mass of unit volume, respectively. $e_{0}$ is atomic material constant and $a$ is characteristic internal length. Also, $u$ means the axial motion. Total partial integration of the average weighted residue can be reached:

$$
\int_{0}^{L}\left[-E A \frac{\partial h}{\partial x} \frac{\partial u}{\partial x}-\rho A h \frac{\partial^{2} u}{\partial t^{2}}-\left(e_{0} a\right)^{2} \rho A \frac{\partial h}{\partial x} \frac{\partial^{3} u}{\partial x \partial t^{2}}\right] \mathrm{d} x=0
$$

In order to rearrange Eq. (5), the axial motion and its kinematic relation should be defined:

$$
u=\phi \mathbf{u}, \frac{\partial u}{\partial x}=\mathrm{D}^{\mathrm{k}} u=\mathbf{B u}, \frac{\partial^{2} u}{\partial t^{2}}=\phi \ddot{\mathbf{u}}
$$

where $\mathbf{u}$ is axial motion vector of end freedoms of longitudinal element. Additionally, $\mathrm{D}^{\mathrm{k}}$ is kinematic operator ( $D^{k} \phi=\mathbf{B}$ ). After Eq. (6) is replaced into Eq. (5), the following definitions can be made:

$$
\begin{gathered}
K=\int_{0}^{L} E A\left(\mathbf{B}^{\mathrm{T}} \mathbf{B}\right) \mathrm{d} x=\frac{E A}{L}\left[\begin{array}{cc}
1 & -1 \\
-1 & 1
\end{array}\right] \\
M_{c}=\int_{0}^{L} \rho A\left(\phi^{\mathrm{T}} \phi\right) \mathrm{d} x=\frac{\rho A L}{6}\left[\begin{array}{ll}
2 & 1 \\
1 & 2
\end{array}\right] \\
M_{n l}=\int_{0}^{L}\left(e_{0} a\right)^{2} \rho A\left(\mathbf{B}^{\mathrm{T}} \mathbf{B}\right) \mathrm{d} x=\frac{\left(e_{0} a\right)^{2} \rho A}{L}\left[\begin{array}{cc}
1 & -1 \\
-1 & 1
\end{array}\right]
\end{gathered}
$$

where $K$ is axial stiffness matrix. $M_{c}$ and $M_{n l}$ explain the classical and nonlocal mass matrices, respectively.

\subsection{Bending Vibration}

The equation of motion of nonlocal free bending vibration is expressed as [30]:

$$
E I \frac{\partial^{4} w}{\partial x^{4}}+\rho A \frac{\partial^{2} w}{\partial t^{2}}+\left(e_{0} a\right)^{2} \rho A \frac{\partial^{4} w}{\partial x^{2} \partial t^{2}}=0
$$

In which, $I$ is moment of inertia and $w$ is transverse motion. Similar to axial vibration, the average weighted residue result is rewritten as follow:

$$
\int_{0}^{L}\left[-E I \frac{\partial^{2} h}{\partial x^{2}} \frac{\partial^{2} w}{\partial x^{2}}-\rho A h \frac{\partial^{2} w}{\partial t^{2}}-\left(e_{0} a\right)^{2} \rho A \frac{\partial h}{\partial x} \frac{\partial^{3} w}{\partial x \partial t^{2}}\right] \mathrm{d} x=0
$$


Also, transverse motion and its kinematic relations are presented as:

$$
w=\phi \mathbf{w}, \frac{\partial w}{\partial x}=\mathrm{D}^{\mathrm{k}} w=\mathbf{B} \mathbf{w}, \frac{\partial^{2} w}{\partial x^{2}}=\mathbf{B}^{\prime} \mathbf{w}, \frac{\partial^{2} w}{\partial t^{2}}=\phi \ddot{\mathbf{w}}
$$

Substituting Eq. (12) into Eq. (11) yields the following bending stiffness matrix $K$, classical mass matrix $M_{c}$, and nonlocal mass matrix $M_{n l}$, respectively [26]:

$$
\begin{gathered}
K=\int_{0}^{L} E A\left(\mathbf{B}^{\prime \mathrm{T}} \mathbf{B}^{\prime}\right) \mathrm{d} x=\frac{E I}{L^{3}}\left[\begin{array}{cccc}
12 & 6 L & -12 & 6 L \\
6 L & 4 L^{2} & -6 L & 2 L^{2} \\
-12 & -6 L & 12 & -6 L \\
6 L & 2 L^{2} & -6 L & 4 L^{2}
\end{array}\right] \\
M_{c}=\int_{0}^{L} \rho A\left(\phi^{\mathrm{T}} \phi\right) \mathrm{d} x=\frac{\rho A L}{420}\left[\begin{array}{cccc}
156 L & 22 L^{2} & 54 L & -13 L^{2} \\
22 L^{2} & 4 L^{3} & 13 L^{2} & -3 L^{3} \\
54 L & 13 L^{2} & 156 L & -22 L^{3} \\
-13 L & -3 L^{3} & -22 L^{2} & 4 L^{3}
\end{array}\right] \\
M_{n l}=\int_{0}^{L}\left(e_{0} a\right)^{2} \rho A\left(\mathbf{B}^{\mathrm{T}} \mathbf{B}\right) \mathrm{d} x=\frac{\left(e_{0} a\right)^{2} \rho A}{30 L}\left[\begin{array}{cccc}
36 & 3 L & -36 & 3 L \\
3 L & 4 L^{2} & -3 L & -L^{2} \\
-36 & -3 L & 36 & -3 L \\
3 L & -L^{2} & -3 L & 4 L^{2}
\end{array}\right]
\end{gathered}
$$

\section{Nonlocal Matrix Displacement Formulation}

To constitute the vibration formulation of the structures consisting of discrete members, the stiffness and mass matrices of the element that makes a positive $\alpha$ angle with the horizontal in the general axes should be determined. The detail of this process can be found in [26]. Accordingly, the stiffness and mass matrices are transformed from the global axes to the local axes.

The transformation matrices for discrete structures under axial effects only (nanotrusses) and both axial and bending effects (nanoframes) are as follows, respectively:

$$
T_{a}=\left[\begin{array}{cccc}
\cos \alpha & \sin \alpha & 0 & 0 \\
-\sin \alpha & \cos \alpha & 0 & 0 \\
0 & 0 & \cos \alpha & \sin \alpha \\
0 & 0 & -\sin \alpha & \cos \alpha
\end{array}\right]
$$




$$
T_{b}=\left[\begin{array}{cccccc}
\cos \alpha & \sin \alpha & 0 & 0 & 0 & 0 \\
-\sin \alpha & \cos \alpha & 0 & 0 & 0 & 0 \\
0 & 0 & 1 & 0 & 0 & 0 \\
0 & 0 & 0 & \cos \alpha & \sin \alpha & 0 \\
0 & 0 & 0 & -\sin \alpha & \cos \alpha & 0 \\
0 & 0 & 0 & 0 & 0 & 1
\end{array}\right]
$$

\subsection{Nonlocal Matrices of Nanotrusses}

Since the axial discrete member has not a stiffness in the perpendicular direction to the element, the inputs of the matrix in Eq. (7) constitute only the axial freedoms of discrete member. However, due to Newton's second law, since the mass constitute the acceleration of the motion in the axial and transverse freedoms, the matrices calculated in Eqs. (8) and (9) determine the inputs of discrete member in both axial both and transverse directions [26].

The stiffness and mass matrices in the local axes are calculated as follows [26]:

$$
\begin{aligned}
& K_{e}=T_{a}^{\mathrm{T}} K T_{a}= \\
& {\left[\begin{array}{cccc}
\cos \alpha & -\sin \alpha & 0 & 0 \\
\sin \alpha & \cos \alpha & 0 & 0 \\
0 & 0 & \cos \alpha & -\sin \alpha \\
0 & 0 & \sin \alpha & \cos \alpha
\end{array}\right] \times \frac{E A}{L}\left[\begin{array}{cccc}
1 & 0 & -1 & 0 \\
0 & 0 & 0 & 0 \\
-1 & 0 & 1 & 0 \\
0 & 0 & 0 & 0
\end{array}\right] \times\left[\begin{array}{cccc}
\cos \alpha & \sin \alpha & 0 & 0 \\
-\sin \alpha & \cos \alpha & 0 & 0 \\
0 & 0 & \cos \alpha & \sin \alpha \\
0 & 0 & -\sin \alpha & \cos \alpha
\end{array}\right]} \\
& =\frac{E A}{L}\left[\begin{array}{cccc}
\cos ^{2} \alpha & \cos \alpha \sin \alpha & -\cos ^{2} \alpha & -\cos \alpha \sin \alpha \\
\cos \alpha \sin \alpha & \sin ^{2} \alpha & -\cos \alpha \sin \alpha & -\sin ^{2} \alpha \\
-\cos ^{2} \alpha & -\cos \alpha \sin \alpha & \cos ^{2} \alpha & \cos \alpha \sin \alpha \\
-\cos \alpha \sin \alpha & -\sin ^{2} \alpha & \cos \alpha \sin \alpha & \sin ^{2} \alpha
\end{array}\right] \\
& M_{e}=T_{a}^{\mathrm{T}}\left(M_{c}+M_{n l}\right) T_{a}= \\
& {\left[\begin{array}{cccc}
\cos \alpha & -\sin \alpha & 0 & 0 \\
\sin \alpha & \cos \alpha & 0 & 0 \\
0 & 0 & \cos \alpha & -\sin \alpha \\
0 & 0 & \sin \alpha & \cos \alpha
\end{array}\right] \times\left(\frac{\rho A L}{6}\left[\begin{array}{cccc}
2 & 0 & 1 & 0 \\
0 & 2 & 0 & 1 \\
1 & 0 & 2 & 0 \\
0 & 1 & 0 & 2
\end{array}\right]+\frac{\left(e_{0} a\right)^{2} \rho A}{L}\left[\begin{array}{cccc}
1 & 0 & -1 & 0 \\
0 & 1 & 0 & -1 \\
-1 & 0 & 1 & 0 \\
0 & -1 & 0 & 1
\end{array}\right]\right) \times} \\
& {\left[\begin{array}{cccc}
\cos \alpha & \sin \alpha & 0 & 0 \\
-\sin \alpha & \cos \alpha & 0 & 0 \\
0 & 0 & \cos \alpha & \sin \alpha \\
0 & 0 & -\sin \alpha & \cos \alpha
\end{array}\right]=\rho A L\left[\begin{array}{ccccc}
\frac{1}{3}+\frac{\left(e_{0} a\right)^{2}}{L^{2}} & 0 & \frac{1}{3}+\frac{\left(e_{0} a\right)^{2}}{L^{2}} & 0 \\
0 & \frac{1}{3}+\frac{\left(e_{0} a\right)^{2}}{L^{2}} & 0 & \frac{1}{6}-\frac{\left(e_{0} a\right)^{2}}{L^{2}} \\
\frac{1}{6}-\frac{\left(e_{0} a\right)^{2}}{L^{2}} & 0 & \frac{1}{3}+\frac{\left(e_{0} a\right)^{2}}{L^{2}} & 0 \\
0 & \frac{1}{3}+\frac{\left(e_{0} a\right)^{2}}{L^{2}} & 0 & \frac{1}{3}+\frac{\left(e_{0} a\right)^{2}}{L^{2}}
\end{array}\right]}
\end{aligned}
$$


The vibration of the nanotruss is solved by the eigenvalue formulation as follow:

$$
\operatorname{det}\left(\sum_{i=1}^{n e}\left[K_{e}\right]_{n \times n}^{*}-\omega_{n}^{2} \sum_{i=1}^{n e}\left[M_{e}\right]_{n \times n}^{*}\right)=0
$$

where $\left[K_{e}\right]^{*}$ and $\left[M_{e}\right]^{*}$ represent the reduced total stiffness and mass matrices, respectively. Also, $n$ is degree of freedom of discrete system. $\omega_{n}$ denotes the natural frequency.

\subsection{Nonlocal Matrices of Nanoframes}

Because bending discrete members have freedoms in the axial direction, too, the stiffness and mass matrices in the global axes can be assembled by considering Eqs. (7)-(9) and (13)-(15) [26]:

$$
K=\left[\begin{array}{cccccc}
\frac{E A}{L} & 0 & 0 & -\frac{E A}{L} & 0 & 0 \\
0 & \frac{12 E I}{L^{3}} & -\frac{6 E I}{L^{2}} & 0 & -\frac{12 E I}{L^{3}} & -\frac{6 E I}{L^{2}} \\
0 & -\frac{6 E I}{L^{2}} & \frac{4 E I}{L} & 0 & \frac{6 E I}{L^{2}} & \frac{2 E I}{L} \\
-\frac{E A}{L} & 0 & 0 & \frac{E A}{L} & 0 & 0 \\
0 & -\frac{12 E I}{L^{3}} & \frac{6 E I}{L^{2}} & 0 & \frac{12 E I}{L^{3}} & \frac{6 E I}{L^{2}} \\
0 & -\frac{6 E I}{L^{2}} & \frac{2 E I}{L} & 0 & \frac{6 E I}{L^{2}} & \frac{4 E I}{L}
\end{array}\right]
$$




$$
M_{n l}=\left(e_{0} a\right)^{2} \rho A L\left[\begin{array}{cccccc}
\frac{1}{L} & 0 & 0 & -\frac{1}{L} & 0 & 0 \\
0 & \frac{6}{5 L} & \frac{1}{10} & 0 & -\frac{6}{5 L} & \frac{1}{10} \\
0 & \frac{1}{10} & \frac{2 L}{15} & 0 & -\frac{1}{10} & -\frac{L}{30} \\
-\frac{1}{L} & 0 & 0 & \frac{1}{L} & 0 & 0 \\
0 & -\frac{6}{5 L} & -\frac{1}{10} & 0 & \frac{6}{5 L} & -\frac{1}{10} \\
0 & \frac{1}{10} & -\frac{L}{30} & 0 & -\frac{1}{10} & \frac{2 L}{15}
\end{array}\right]
$$

The stiffness and mass matrices of the bending discrete member are expressed as:

$$
\begin{gathered}
K_{e}=T_{b}^{\mathrm{T}} K T_{b}=\left[k_{i j}\right]_{6 \times 6} \\
M_{e}=T_{b}^{\mathrm{T}}\left(M_{c}+M_{n l}\right) T_{b}=\left[m_{i j}\right]_{6 \times 6}
\end{gathered}
$$

The components of the stiffness matrix are calculated as follows:

$$
\begin{gathered}
k_{11}=k_{44}=\frac{E A}{L} \cos ^{2} \alpha+\frac{12 E I}{L^{3}} \sin ^{2} \alpha, k_{12}=k_{45}=\left(\frac{E A}{L}-\frac{12 E I}{L^{3}}\right) \cos \alpha \sin \alpha, \\
k_{13}=k_{16}=-\frac{6 E I}{L^{2}} \sin \alpha, k_{14}=-\frac{E A}{L} \cos ^{2} \alpha-\frac{12 E I}{L^{3}} \sin ^{2} \alpha, \\
k_{15}=k_{24}=\left(-\frac{E A}{L}+\frac{12 E I}{L^{3}}\right) \cos \alpha \sin \alpha, k_{22}=k_{55}=\frac{E A}{L} \sin ^{2} \alpha+\frac{12 E I}{L^{3}} \cos ^{2} \alpha, \\
k_{23}=k_{26}=\frac{6 E I}{L^{2}} \mathrm{c}, k_{25}=-\frac{E A}{L} \sin ^{2} \alpha-\frac{12 E I}{L^{3}} \cos ^{2} \alpha, k_{33}=k_{66}=\frac{4 E I}{L}, \\
k_{34}=k_{46}=\frac{6 E I}{L^{2}} \sin \alpha, k_{35}=k_{56}=-\frac{6 E I}{L^{2}} \cos \alpha, k_{36}=\frac{2 E I}{L}
\end{gathered}
$$

Additionally, the components of the mass matrix can be given as:

$$
\begin{gathered}
m_{11}=m_{44}=\left(\frac{\rho A L}{3}+\frac{\left(e_{0} a\right)^{2} \rho A}{L}\right) \cos ^{2} \alpha+\left(\frac{13 \rho A L}{35}+\frac{6\left(e_{0} a\right)^{2} \rho A}{5 L}\right) \sin ^{2} \alpha, \\
m_{12}=m_{45}=\left(-\frac{4 \rho A L}{105}-\frac{\left(e_{0} a\right)^{2} \rho A}{5 L}\right) \cos \alpha \sin \alpha, m_{13}=\left(-\frac{11 \rho A L^{2}}{210}-\frac{\left(e_{0} a\right)^{2} \rho A}{10}\right) \sin \alpha, \\
m_{14}=\left(\frac{\rho A L}{6}-\frac{\left(e_{0} a\right)^{2} \rho A}{L}\right) \cos ^{2} \alpha+\left(\frac{9 \rho A L}{70}-\frac{6\left(e_{0} a\right)^{2} \rho A}{5 L}\right) \sin ^{2} \alpha, \\
m_{15}=m_{24}=\left(\frac{4 \rho A L}{105}+\frac{\left(e_{0} a\right)^{2} \rho A}{5 L}\right) \cos \alpha \sin \alpha, m_{16}=\left(\frac{13 \rho A L^{2}}{420}-\frac{\left(e_{0} a\right)^{2} \rho A}{10}\right) \sin \alpha,
\end{gathered}
$$




$$
\begin{gathered}
m_{22}=m_{55}=\left(\frac{\rho A L}{3}+\frac{6\left(e_{0} a\right)^{2} \rho A}{5 L}\right) \cos ^{2} \alpha+\left(\frac{13 \rho A L}{35}+\frac{\left(e_{0} a\right)^{2} \rho A}{L}\right) \sin ^{2} \alpha \\
m_{23}=\left(\frac{11 \rho A L^{2}}{210}+\frac{\left(e_{0} a\right)^{2} \rho A}{10}\right) \cos \alpha, \\
m_{25}=\left(\frac{9 \rho A L}{70}-\frac{6\left(e_{0} a\right)^{2} \rho A}{5 L}\right) \cos ^{2} \alpha+\left(\frac{\rho A L}{6}-\frac{\left(e_{0} a\right)^{2} \rho A}{L}\right) \sin ^{2} \alpha, \\
m_{26}=\left(-\frac{13 \rho A L^{2}}{420}+\frac{\left(e_{0} a\right)^{2} \rho A}{10}\right) \cos \alpha, \quad m_{33}=m_{66}=\frac{\rho A L^{3}}{105}+\frac{2\left(e_{0} a\right)^{2} \rho A L}{15}, \\
m_{34}=\left(-\frac{13 \rho A L^{2}}{420}+\frac{\left(e_{0} a\right)^{2} \rho A}{10}\right) \sin \alpha, \quad m_{35}=\left(\frac{13 \rho A L^{2}}{420}-\frac{\left(e_{0} a\right)^{2} \rho A}{10}\right) \cos \alpha, \\
m_{36}=-\frac{\rho A L^{3}}{140}-\frac{\left(e_{0} a\right)^{2} \rho A L}{30}, m_{46}=\left(\frac{11 \rho A L^{2}}{210}+\frac{\left(e_{0} a\right)^{2} \rho A}{10}\right) \sin \alpha \\
m_{56}=\left(-\frac{11 \rho A L^{2}}{210}-\frac{\left(e_{0} a\right)^{2} \rho A}{10}\right) \cos \alpha
\end{gathered}
$$

Free vibration frequencies of nanoframes are also calculated as in Eq. (20).

\section{Discussions}

The solution based on matrix displacement method for the nonlocal free dynamics of nano scaled truss and frame structures is mentioned. According to this, the stiffness and mass matrices of the nanostructure members are achieved. When obtained expressions are investigated, it is understood that the stiffness matrices are not affected by the nonlocal parameter, and additionally, the nonlocal parameter is included in the inputs of the mass matrix. The reason for this is the nonlocal parameter is only added as a multiplier to the mass of unit length $(\rho A)$ in the equations of motion which the average weighted residue is applied.

\section{Conclusions}

While studies dealing with the nonlocal mechanics of nanostructures with continuous system models such as beams and rods are numerous, studies on the mechanical analysis of nano scaled structures with discrete models are quite limited. This current study is explained the stiffness and mass matrices in the matrix displacement formulation given for the dynamic analysis of nano scaled trusses and frames are modified how by the nonlocal parameter.

In the nonlocal free dynamic analysis of nanotrusses and nanoframes, the fact that the stiffness matrices are the same as the classical elasticity and the mass matrix increases, shows that the classical natural frequencies will be a decrease due to the nonlocal parameter in the dynamic analysis of the nanostructures consisting of discrete members [26]. Therefore, since the response of the structure to dynamic excitations will decrease, this case should be taken into account in the design of the engineering system where nanostructures may take part. 


\section{References}

[1] Aydogdu, M., Axial vibration of the nanorods with the nonlocal continuum rod model. Physica E: Low-dimensional Systems and Nanostructures, 41, 861-864, 2009.

[2] Adhikari, S., Murmu, T., McCarthy, M.A., Dynamic finite element analysis of axially vibrating nonlocal rods. Finite Elements in Analysis and Design, 630, 42-50, 2013.

[3] Demir, Ç., Civalek, Ö., Torsional and longitudinal frequency and wave response of microtubules based on the nonlocal continuum and nonlocal discrete models. Applied Mathematical Modelling, 37, 9355-9367, 2013.

[4] Lim, C.W., Islam, M.Z., Zhang, G., A nonlocal finite element method for torsional statics and dynamics of circular nanostructures. International Journal of Mechanical Sciences, 94-95, 232-243, 2015.

[5] Zhu, X., Li, L., On longitudinal dynamics of nanorods. International Journal of Engineering Science, 120, 129-145, 2017.

[6] Numanoğlu, H.M., Akgöz, B., Civalek, Ö., On dynamic analysis of nanorods. International Journal of Engineering Science, 130, 33-50, 2018.

[7] Karlicic, D.Z., Ayed, S., Flaieh, E., Nonlocal axial vibration of the multiple Bishop nanorod system. Mathematics and Mechanics of Solids, 24, 1668-1691, 2018.

[8] Nazemnezhad, R., Kamali, K., Free axial vibration analysis of axially functionally graded thick nanorods using nonlocal Bishop's theory. Steel and Composite Structures, 28, 749758, 2018.

[9] Bao, S., Cao, J., Wang, S., Vibration analysis of nanorods by the Rayleigh-Ritz method and truncated Fourier series. Results in Physics, 12, 327-334, 2019.

[10] Numanoğlu, H.M., Civalek Ö., On the torsional vibration of nanorods surrounded by elastic matrix via nonlocal FEM, International Journal of Mechanical Sciences, 161-162, 105076, 2019.

[11] Civalek, Ö., Numanoğlu, H.M., Nonlocal finite element analysis for axial vibration of embedded Love-Bishop nanorods. International Journal of Mechanical Sciences, 188, $105939,2020$.

[12] Reddy, J.N., Nonlocal theories for bending, buckling and vibration of beams. International Journal of Engineering Science, 45, 288-307, 2007.

[13] Benzair, A., Tounsi, A., Besseghier, A., Heireche, H., Moulay, N., Boumia, L., The thermal effect on vibration of single-walled carbon nanotubes using nonlocal Timoshenko beam theory. Journal of Physics D: Applied Physics, 41, 225404, 2008.

[14] Thai, H.T., A nonlocal beam theory for bending, buckling and vibration of nanobeams. International Journal of Engineering Science, 52, 56-64, 2012.

[15] Askari, H., Esmailzadeh, E., Zhang, D., Nonlinear vibration analysis of nonlocal nanowires. Composites Part B: Engineering, 67, 607-613, 2014. 
[16] Mercan, K., Numanoğlu, H.M., Akgöz, B., Demir, C., Civalek, Ö., Higher-order continuum theories for buckling response of silicon carbide nanowires (SiCNWs) on elastic matrix. Archive of Applied Mechanics, 87, 1797-1814, 2017.

[17] Eltaher, M.A., Alshorbagy, A.E., Mahmoud, F.F., Vibration analysis of Euler-Bernoulli nanobeams by using finite element method. Applied Mathematical Modelling, 37, 47874797, 2013.

[18] Eptaimeros, K.G., Koutsoumaris, C.C., Tsamasphyros, G.J., Nonlocal integral approach to the dynamical response of nanobeams. International Journal of Mechanical Sciences, 115116, 68-80, 2016.

[19] Civalek, Ö., Demir, C., A simple mathematical model of microtubules surrounded by an elastic matrix by nonlocal finite element method. Applied Mathematics and Computation, 289, 335-352, 2016.

[20] Numanoğlu, H.M., Uzun, B., Civalek, Ö., Derivation of nonlocal finite element formulation for nano beams. International Journal of Engineering and Applied Sciences, 10, 131-139, 2018.

[21] Numanoğlu, H.M., Mercan, K, Civalek, Ö., Finite element model and size-dependent stability analysis of boron nitride and silicon carbide nanowires/nanotubes. Scientia Iranica Transactions A: Civil Engineering, 26, 2079-2099, 2019.

[22] Numanoğlu, H.M., Thermal vibration of zinc oxide nanowires by using nonlocal finite element method. International Journal of Engineering and Applied Sciences, 12, 99-110, 2020.

[23] Numanoğlu, H.M., Ersoy, H., Civalek, O., Ferreira, A.J.M., Derivation of nonlocal FEM formulation for thermo-elastic Timoshenko beams on elastic matrix. Composite Structures, 273, 114292, 2021.

[24] Ersoy, H, Numanoğlu, H.M., Akgöz, B., Civalek Ö., A new eigenvalue problem solver for thermo-mechanical vibration of Timoshenko nanobeams by an innovative nonlocal finite element method. Mathematical Methods in the Applied Sciences, 2021.

[25] de Sciarra, F.M., Finite element modelling of nonlocal beams. Physica E: Low-Dimesional Systems and Nanostructures, 59, 144-149, 2014.

[26] Numanoğlu, H.M., Civalek Ö., On the dynamics of small-sized structures, International Journal of Engineering Science, 145, 103164, 2019.

[27] Hozhabrossadati, S.M., Challamel, N., Rezaiee-Pajand, M., Sani, A.A., Free vibration of a nanogrid based on Eringen's stress gradient model. Mechanics Based Design of Structures and Machines, 2020.

[28] Russillo, A.F., Failla, G., Alotta, G., de Sciarra, F.M., Barretta, R., On the dynamics of nano-frames. International Journal of Engineering Science, 160, 103433, 2021.

[29] Numanoğlu, H.M., Mercan, K., Civalek, Ö., Frequency and mode shapes of Au nanowires using the continuous beam models. International Journal of Engineering and Applied Sciences, 9, 55-61, 2017. 
[30] Numanoğlu, H.M., Nanoyapıların Kiriş ve Çubuk Modellerinin Yerel Olmayan Elastisite Teorisi Kullanılarak Titreşim Analizi. BSc. Thesis, Akdeniz University, Antalya, 2017.

[31] Numanoğlu, H.M., Civalek, Ö., Elastic beam model and bending analysis of silver nanowires. International Journal of Engineering and Applied Sciences, 10, 13-20, 2018.

[32] Numanoğlu, H.M., Ersoy, H, Akgöz, B., Civalek Ö., Small size and rotary inertia effects on the natural frequencies of carbon nanotubes. Curved and Layered Structures, 5, $273-$ $279,2018$.

[33] Akgöz, B., Civalek Ö., Vibrational characteristics of embedded microbeams lying on a two-parameter elastic foundation in thermal environment. Composites Part B: Engineering, 150, 68-77, 2018.

[34] Akgöz, B., Civalek, Ö., Investigation of size effects on static response of single-walled carbon nanotubes based on strain gradient elasticity. International Journal of Computational Methods, 9, 1240032, 2012.

[35] Civalek, Ö., Kiracioglu, O., Free vibration analysis of Timoshenko beams by DSC method. International Journal for Numerical Methods in Biomedical Engineering, 26, 1890-1898, 2010.

[36] Mercan, K., Demir, Ç., Civalek, Ö., Vibration analysis of FG cylindrical shells with powerlaw index using discrete singular convolution technique. Curved and Layered Structures, 3, 82-90, 2016.

[37] Dastjerdi, S., Akgöz, B., Civalek, Ö., On the effect of viscoelasticity on behavior of gyroscopes. International Journal of Engineering Science, 149, 103236, 2020.

[38] Civalek, Ö., Dastjerdi, S., Akbaş, Ş.D., Akgöz, B., Vibration analysis of carbon nanotubereinforced composite microbeams. Mathematical Methods in the Applied Sciences, 2021.

[39] Dastjerdi, S., Akgöz, B., New static and dynamic analyses of macro and nano FGM plates using exact three-dimensional elasticity in thermal environment. Composite Structures, 192, 626-641, 2018.

[40] Uzun, B, Numanoğlu, H.M., Civalek, Ö., Definition of length-scale parameter in Eringen's nonlocal elasticity via nolocal lattice and finite element formulation. International Journal of Engineering and Applied Sciences, 10, 264-275, 2018.

[41] Numanoğlu, H.M., Dynamic analysis of nano continuous and discrete structures based on nonlocal finite element formulation (NL-FEM). MSc. Thesis, Akdeniz University, Antalya, 2019, (In Turkish). 